\title{
The Optical Properties of Indocyanine Green suspended in Solution as Observed under Near Infrared LED and LASER Light Conditions
}

\author{
Angharad Curtis \\ Wireless \& \\ Optoelectronics \\ Research and \\ Innovation Centre, \\ University of South \\ Wales \\ Pontypridd, Wales, \\ UK
}

\author{
Kang Li \\ Wireless \& \\ Optoelectronics \\ Research and \\ Innovation Centre, \\ University of South \\ Wales \\ Pontypridd, Wales, \\ UK
}

\author{
Mohammed Ali \\ Roula \\ School of \\ Engineering, \\ University of South \\ Wales \\ Pontypridd, Wales, \\ UK
}

\author{
Nigel Copner \\ Wireless \& \\ Optoelectronics \\ Research and \\ Innovation Centre, \\ University of South \\ Wales \\ Pontypridd, Wales, \\ UK
}

\begin{abstract}
The use of Indocyanine Green (ICG) as a fluorescent marker at Near Infrared (NIR) excitation wavelengths is well established in clinical imaging. Typical systems comprise multiple LED sources for optimal imaging which can result in unnecessary energy transfer to patients and contribute to tissue damage. An experimental setup comprising a $780 \mathrm{~nm}$ excitation channel generating up to $10 \mathrm{~mW}$ of optical power is used in order to determine if there is potential to exploit the optical properties of ICG, in order to reduce the total excitation power through pulsing. We demonstrate in this work that a single 1.6 Megapixel CMOS camera with quantum efficiency of less than $30 \%$ is appropriate to capture both fluorescent and non-fluorescent landmarks at NIR wavelengths. Experimental results verify that all ICG solutions tested yielded detectable fluorescence and that degradation of fluorescence intensity over time is multifaceted.
\end{abstract}

Keywords: Fluorescence Imaging, Indocyanine Green (ICG), Fluorescence Endoscopy, In-Vitro Testing, Fluorescence Spectrometry

\begin{abstract}
1. INTRODUCTION
There are a number of detailed reviews in the literature related to the fluorescent chemical marker Indocyanine Green (ICG) and its importance in clinical imaging [1,2]. ICG is excited with either LED, Xenon or LASER light at NIR wavelengths causing fluorescence. This essentially illuminates cells which have absorbed and retained the fluorescent marker during minimally invasive surgery, allowing for a more efficient and complete resection of diseased tissue. ICG is currently one of two FDA approved dyes at this wavelength range [3] and has been approved for medical use since the 50's [1].
\end{abstract}

The fluorescence decay rate and photobleaching properties of ICG for fluorescence endoscopy are not well documented but are crucial to understand. The literature related to the photobleaching effect of ICG appears conflicting [4-6] and there is currently no standardization regarding test procedures and no consistency in findings. If the optical proper-ties are identified, there could be opportunity to exploit them so that ICG usage in general can be optimized resulting in enhanced patient safety.

Typically, when using ICG as an identifying marker for diseased tissue, a white light channel is required in order to view non-fluorescent/unmarked landmarks within the frame such as blood vessels. The NIR and white light channels individually, do not allow for a complete resection of diseased tissue with minimal removal of healthy tissue as the imaging conditions are not optimal and this can prove dangerous [7]. The most successful protocol is to combine data from both channels which involves possible risks and com-plications. The main risk being excessive excitation used thus exposing the patient to a higher risk of thermal damage during minimally invasive surgical conditions

An experimental four channel LED multiplexer [8], awaiting clinical trials, designed to replace the 'light box' component in fluorescence endoscopy focuses red, green blue and near infrared LED light to a liquid light guide. The small aperture of the liquid light guide sets the etendue limit. Heat is produced as a by-product from the illumination source and can become intense causing permanent damage to tissue [9, 10]. Optical power of the NIR source needs to reach the desired penetration depth without adding to the unwanted thermal output [11]. Optical power used in this research is between $5 \mathrm{~mW}$ and $10 \mathrm{~mW}$ in agreement with relevant work in the literature [12-14]. The literature also suggests that excitation could be pulsed to reduce the thermal effects [15].

In this work we demonstrate the viability of a system comprising a pulsed excitation channel and a single CMOS detector with the aim to image ICG-stained samples using an inexpensive camera in order to capture residual fluorescence and determine the relation-ship between thermal decay and photobleaching. While this work relates to an endoscopy application for the detection of cancerous tissue in the breast using ICG as a chemical marker, the experimental work is conducted in a laboratory environment using a wide-field approach for various reasons.

\section{MATERIAL AND METHODS}

Samples of various concentrations of ICG solution were prepared for this experimental research. The ICG (Tokyo Chemical Industry UK Ltd) is mixed with Human Serum Albumin (HSA) in order to enhance stability and fluorescence $[12-13,16]$. The samples are il-luminated with either LED or LASER excitation at $780 \mathrm{~nm}$ (Thorlabs L780P010 and Thorlabs LED780E respectively) in order to excite the chemical marker causing it to fluoresce. This fluorescence is detected using a spectrometer (Ocean Optics USB4000) and captured via a CMOS camera with signal to noise ratio of $40.48 \mathrm{~dB}$. Data collected is processed to determine if it is possible to detect any residual fluorescence at the instant 
excitation is removed as well as confirming the decay rate of fluorescence and the parameters contributing to this.

\subsection{ICG Sample Preparation}

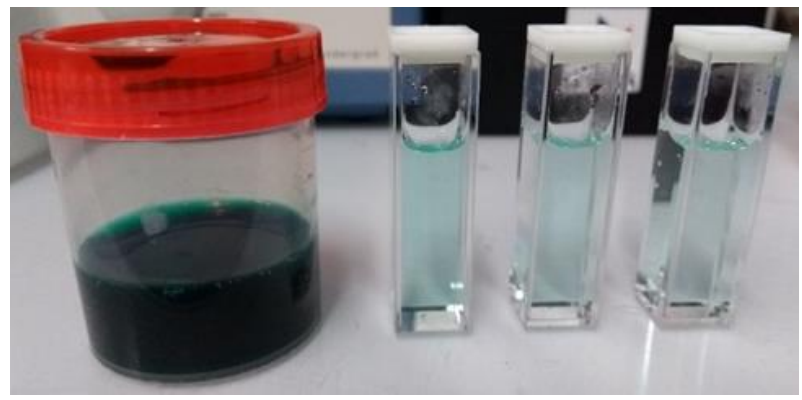

Figure 1: An image showing the ICG stock solution and diluted samples in cuvettes

ICG and HSA (PAN Biotech UK Ltd) are mixed in a 1:1 molar ratio consistent with relevant literature [12, 15-16]. A $1000 \mu \mathrm{Mol}$ solution was prepared allowing for a sufficient dilution level for the desired range of concentrations analyzed. In order to prepare $30 \mathrm{ml}$ of stock solution at a concentration of 1x10-4 Mol, $111.7 \mathrm{mg}$ of HSA and $23.2 \mathrm{mg}$ ICG are dissolved in $30 \mathrm{ml}$ deionized water. This solution is diluted down to $1000 \mu \mathrm{Mol}$ or $1 \times 10-6 \mathrm{Mol}$ by pipetting $0.10 \mathrm{ml}$ of stock solution into $9.90 \mathrm{ml}$ of deionized water. A solution is prepared daily and kept under dark conditions. The temperature of the ambient environment is monitored throughout using a thermometer fitted with an external probe and recorded frequently. There was no significant change in ambient temperature to note. Figure 1 shows the stock solution stored in a plastic container alongside three cuvettes filled with diluted samples of varying concentration. The stock solution does not fluoresce and is the lowest concentration of ICG:HSA achievable with the available apparatus. It is essential to use a transparent mixing vessel to prepare the sample to ensure that the dry constituents are dissolved however, this is kept covered to protect the sample from ultraviolet (UV) degradation.

\subsection{Experimental Setup}

Two similarly arranged systems are assembled for this experiment. One comprises a LA-SER (insert A) light source while the other utilizes a single LED (insert B) as shown in Fig-ure 2. Both the LASER and LED emit light at a central wavelength of $780 \mathrm{~nm}$. The output power of the LASER is manually set to either $5 \mathrm{~mW}$ or $10 \mathrm{~mW}$ by adjusting the current in $\mathrm{mA}$. A calibration curve is plotted to convert the corresponding current to output power by reading the results from a power meter (Thorlabs PM100A fitted with a S302C Head) while adjusting the current in small increments. Once the power threshold has been met, the relationship between current and optical power is linear. A simple circuit is setup to control the LED providing $1.75 \mathrm{~V}$ and comprising an LED controller unit (Gardasoft CC320) for computer controlled on/off and pulsing. Forward optical power for the LED is documented as $7.2 \mathrm{~mW}$ within the technical specifications which corresponds with the power meter readings taken from the perspective and positioning of the cuvette. Table 1 shows the subsequent power at the surface of the sample.
Table 1: Power conversion for each illumination condition

\begin{tabular}{|c|c|c|}
\hline $\begin{array}{c}\text { Illumination } \\
\text { Source }\end{array}$ & $\begin{array}{c}\text { Power } \\
(\mathbf{m W})\end{array}$ & $\begin{array}{c}\text { Power at the } \\
\text { sample } \\
\text { surface } \\
\text { (mW/cm-2) }\end{array}$ \\
\hline LED & 7.2 & 36.67 \\
\hline LASER & 5.0 & 70.74 \\
\hline LASER & 10.0 & 141.47 \\
\hline
\end{tabular}

For continuity, key apparatus such as the spectrometer, power meter, thermometer and CMOS camera are disconnected and swapped out as and when needed so that the optical stages and mounts can remain fixed. The camera used in a BlackFly 16S2M CMOS which is powered and controlled via a USB 3 port, the quantum efficiency of the incorporated sensor (SONY IMX273) at $800 \mathrm{~nm}$ is around $26 \%$. The camera resolution is $1440 \times 1080$ and the pixel size is $3.45 \mu \mathrm{m}$. The power meter detector and the fiber end of the spectrometer can interfere with imaging due to reflection, so these are arranged in a flexible mode whereby they are simply set out of the frame when unused. Two thermometers with ex-ternal probes are also used during data collection. One is used to monitor the temperature of the ambient environment while the other can be placed inside the cuvette and sub-merged in its liquid contents. All experiments are completed under dark conditions with the analysis post data acquisition. The insert $\mathrm{C}$ in Figure 2 shows a schematic of the sample as seen from the perspective of the CMOS camera which is arranged perpendicular to the excitation source. (1) contains the liquid sample only, (2) contains the liquid sample with the probe end of the thermometer in position and (3) shows the fiber end of the spectrometer visible through the liquid contents. Images are not collected under condition (2) or (3) from the figure due to the unwanted reflection caused from the surface of the temperature probe and the spectrometer fiber respectively.

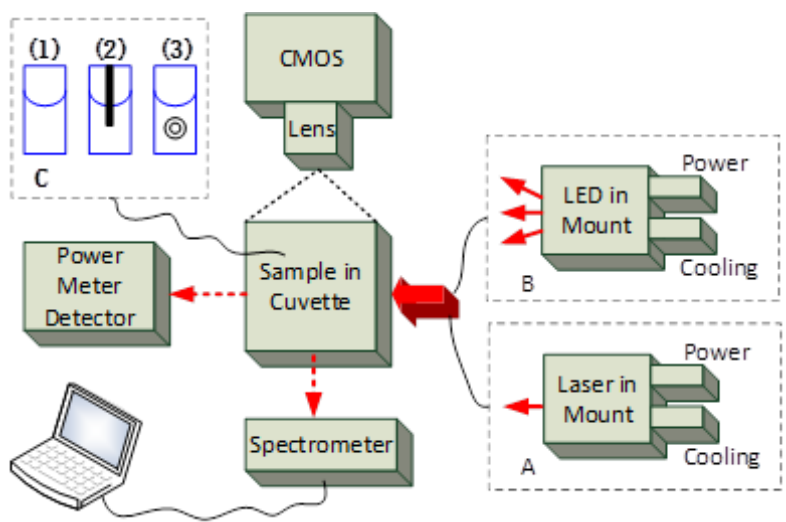

Figure 2: A schematic showing the arrangement of apparatus used during testing (not drawn to scale). Part A features a LASER diode as the excitation source while part B uses a single LED. All other equipment is swapped between the two arrangements. 
Although $780 \mathrm{~nm}$ is visible to the naked eye, a beam viewer card was used during the LASER setup to ensure that the beam is striking the cuvette at the center of the first surface and exiting the final cuvette surface without distortion. The beam is not collimated but an aspheric lens is used to minimize optical aberration. The spot size, $3 \mathrm{~mm}$ was reflected only with inaccurate placement, no reflection is detected within the cuvette following correct positioning in parallel to the source. As the cuvette is positioned manually, inaccurate positioning can result in reflection and scatter which are visible at the furthest corners of the cuvette with respect to the source. This is evident from the images collected such as that seen in Figure 3 and can be rectified with very gentle twisting of the vessel. The housing of the LED allows for a much simpler aligning to the central face of the cuvette which is achieved with the LED off.

\subsection{Data Collection}

Data collected from the CMOS camera is analyzed in a custom LabVIEW programme utilizing the vision and motion palette. The programme displays an intensity analysis of pixels within a user-controlled Region of Interest (ROI). This ROI is saved to memory to keep the position and size consistent between images and can also be re-sized and repositioned when needed. Data from the images is available in real-time and with units according to the parameter under investigation. These numerical values are entered into a spreadsheet ready for plotting using software visualization and analysis tools. The Lab-VIEW programme allows extraction of various features such as the mean intensity of the region given in grayscale values and the minimum and maximum intensity which provides the contrast available for example, at the border of fluorescence. This can also be useful when looking at the intensity of the fluorescence across the pathlength of the cuvette to understand the effect of absorption of the fluorescent material.

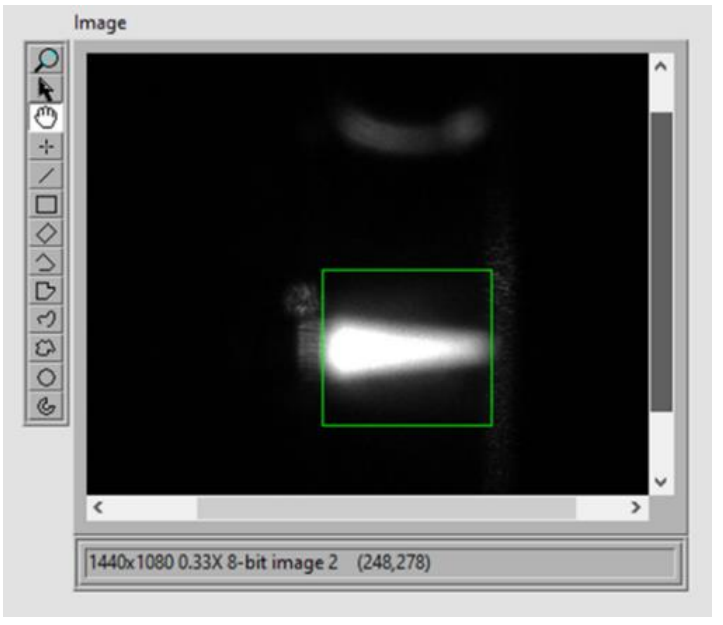

Figure 3: Front end view of the ROI selection of an image showing a $1000 \mu \mathrm{Mol}$ ICG:HSA solution fluo-rescing within a cuvette. The excitation source is a $10 \mathrm{~mW}$ LASER at 780 $\mathrm{nm}$ and exposure time is $249998 \mu \mathrm{s}$. The cuvette walls and meniscus are useful landmarks for analysis and are visible due to scattering.
Spectrometer data is exported from the OceanView software in csv format which is then analyzed. Data collection was repeated six times to enable computation of the mean and standard error. Unfortunately, the spectrometer traces collected show noise across the wavelength range for all repetitions and so a Savitzky-Golay smoothing function was applied to filter out the noise while preserving the shape of the peak. The smoothed spectrometer data is plotted to show the relative intensity of fluorescence for each excitation source and concentration on test. The power meter and temperature data are recorded manually from their respective display interfaces and processed before plotting, pro-cessing again involves calculation of the mean and the corresponding standard error.

The camera frame rate and LED pulses are software controlled. SpinView is used to set the camera frame rate through adjusting the exposure time in microseconds to a minimum of $4855 \mu$ s resulting in a maximum of 199.98 frames per second $\sim 200 \mathrm{~Hz}$. Gardasoft Maintenance software connects the LED controller to the laptop via Ethernet connection. The values are entered manually with a minimum available pulse rate of $0.1 \mathrm{~ms}$. The LED can also be set high (on) or low (off) and the time on phase and time off phase set independently. Timings and pulse rates are recorded in a spreadsheet before each repetition and assigned an alphanumeric code with images saved under a file name with a corresponding code. The SpinView software allows for consecutive images to be collected as either a specified number of frames whereby the frame rate is known or, as a specified number of seconds worth of data capture, again assuming that the frame rate is known and does not exceed the maximum limit of the hardware resulting in dropped frames. Consecutive images can then be analyzed in LabVIEW by simply running through them frame by frame in a similar fashion to if each data set were a video clip. Figure 3 shows the image loaded into LabVIEW with the ROI appearing as the Green boundary. The sides of the cuvette and meniscus of the solution are visible due to scattering which are useful landmarks for analysis.

\section{RESULTS}

Fluorescence data is collected under each excitation condition and the results are presented in Figure 4. Here we can see that the fluorescence wavelength is centered around $815 \mathrm{~nm}, 35$ $\mathrm{nm}$ longer than the excitation. Each figure shows the wavelength range of the spectrometer along the $\mathrm{x}$-axis with the $y$-axis representing the relative intensity of fluorescence in spectrometer units. There are three spectra per figure to correspond with the three excitation conditions. Figure 4 displays the fluorescence spectra detected at each concentration of ICG in solution and illumination condition on test. Fluorescence in-tensity is highest under $10 \mathrm{~mW}$ LASER excitation with the LED producing a more intense fluorescence than the $5 \mathrm{~mW}$ LASER condition. Consistent with literature [13, 18-19], the lowest concentration on test provided the highest fluorescence intensity under each excitation condition, this is due to secondary mechanisms 
related to formation of molecule aggregation. The data presented in Figure 4 represent repeat testing as to avoid errors due to rogue mixing or dilution calculations. The spectrometer collects fluorescence data at intervals of $0.2 \mathrm{~nm}$, an error analysis is displayed in Table 2 representing the peak wave-length value $\sim 818 \mathrm{~nm}$ for simplicity. The power values at $818 \mathrm{~nm}$ are displayed in Figure 4(f).

The reference cuvette containing deionized water shows no fluorescence in Figure 4(e) as expected when excited with the $5 \mathrm{~mW}$ and $10 \mathrm{~mW}$ LASER source. Figure 4(e) shows the LED spectra with a central peak at $780 \mathrm{~nm}$. The reason for this is due to the di-vergence of the LED beam, the light that travels through the cuvette containing water at the excitation wavelength is detected by the spectrometer head. No fluorescence is detected due to the lack of ICG in the sample. As the results presented in Figure 4(e) represent the excitation wavelength of the LED only, we have a better understanding of the "intensity" which accounts for the y-axis of the spectra in Figure 4. The spectrometer and accompanying software define fluorescence as a measurement of the relative irradiance which is not a quantified numerical value. The LED provides just over $7 \mathrm{~mW}$ of forward optical power and so the peak represented by the cuvette containing water only represents a fraction of the source which has travelled through two cuvette walls (optical glass) and a volume of water to reach the detector positioned perpendicular to the source. For reference, the cuvettes have a pathlength of $10 \mathrm{~mm}$ and the glass thickness is $1.25 \mathrm{~mm}$.

Table 2: Error analysis at the fluorescence wavelength peak of $818 \mathrm{~nm}$

\begin{tabular}{|c|c|c|c|}
\hline Solution & $\begin{array}{c}\text { 5mW } \\
\text { LASER } \\
\text { Excitation }\end{array}$ & $\begin{array}{c}\text { 10mW } \\
\text { LASER } \\
\text { Excitation }\end{array}$ & $\begin{array}{c}\text { LED } \\
\text { Excitation }\end{array}$ \\
\hline $400 \mu \mathrm{Mol}$ & $\pm 5.02 \mathrm{~nm}$ & $\pm 5.39 \mathrm{~nm}$ & $\pm 4.1 \mathrm{~nm}$ \\
\hline $600 \mu \mathrm{Mol}$ & $\pm 3.91 \mathrm{~nm}$ & $\pm 3.91 \mathrm{~nm}$ & $\pm 2.43 \mathrm{~nm}$ \\
\hline $800 \mu \mathrm{Mol}$ & $\pm 3.91 \mathrm{~nm}$ & $\pm 3.54 \mathrm{~nm}$ & $\pm 0.96 \mathrm{~nm}$ \\
\hline $1000 \mu \mathrm{Mol}$ & $\pm 2.07 \mathrm{~nm}$ & $\pm 2.07 \mathrm{~nm}$ & $\pm 0.22 \mathrm{~nm}$ \\
\hline
\end{tabular}

To quantify the intensity of the fluorescence, the spectrometer data is used in support of an analysis using images captured with a single CMOS camera. Figure 5(a), (b) and (c) show the maximum, mean and total intensity of the ROI respectively for each concentration and excitation source on test. Images collected are opened in LabVIEW and a region of interest is drawn manually encompassing the contents of the cuvette and excluding the light source. An analysis was conducted in order to obtain the intensity values for pixels within the selected region. These values are plotted and presented to complement the data collected via spectrometry. The CMOS camera images are unfiltered hence the reason for a higher intensity under LED excitation, not observed in the spectrometer results. Figure 5(a) displays the maximum intensity of pixels within the region and Figure 5(b) the mean in-tensity. The upper intensity limit is 256 grayscale units which would saturate the image, consideration of exposure time, excitation power and ICG concentration will yield the most useful images whereby all fluorescent regions are clearly visible without any areas of saturation.

No filtering was applied to the camera setup as confirmed by the spectrometer results. With the exception of water under LED excitation, there is no source wavelength within the results. The camera is in an equivalent position to the spectrometer, in parallel to the source so that the cuvette and its contents are imaged. Images are collected every 60 seconds for 10 minutes at an exposure time of $124998 \mu \mathrm{s}$, a power meter reading is recorded manually at 5 seconds intervals to confirm the camera data and assess the presence of photobleaching or decay in fluorescence over time.

The power meter readings are presented in Figure 6(a), (b) and (c) and the camera data for the $10 \mathrm{~mW}$ excitation with the corresponding camera data are shown in Figure 6(d), (e) and (f). For clarity, no filter has been applied. 
International Journal of Science and Engineering Applications

Volume 10-Issue 05, 80 - 89, 2021, ISSN:- 2319 - 7560

(a)

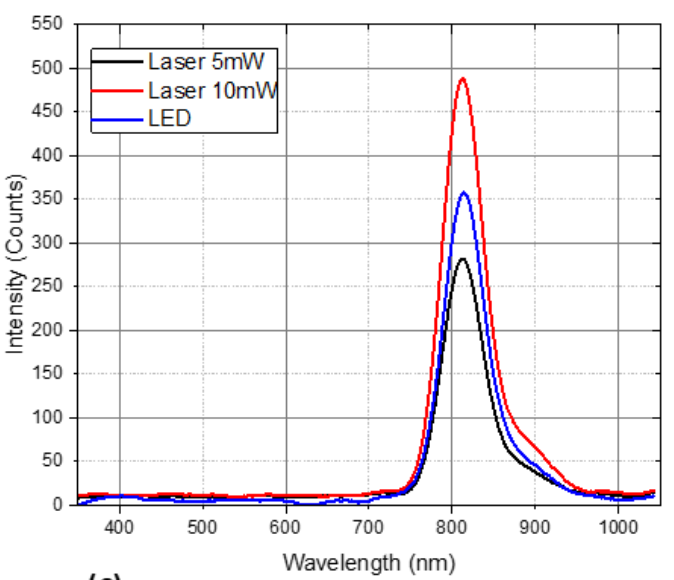

(c)

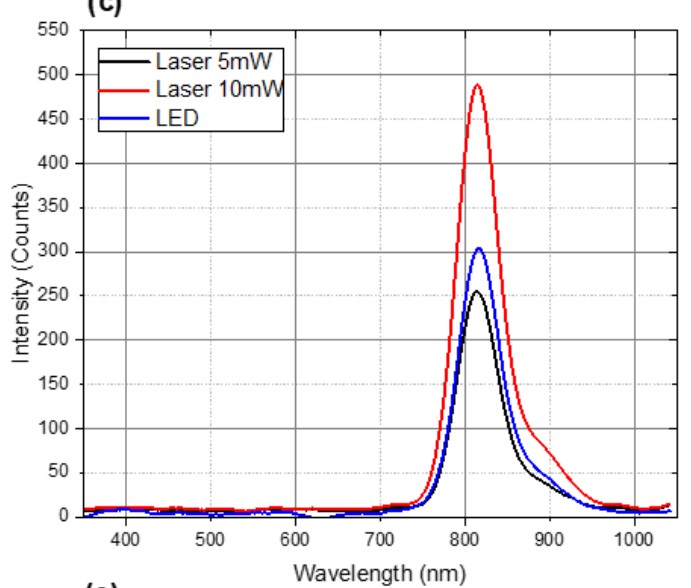

(e)

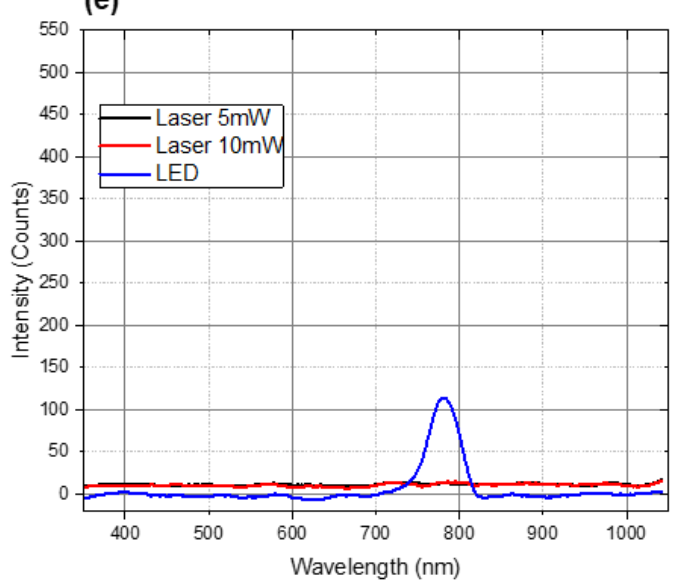

(b)

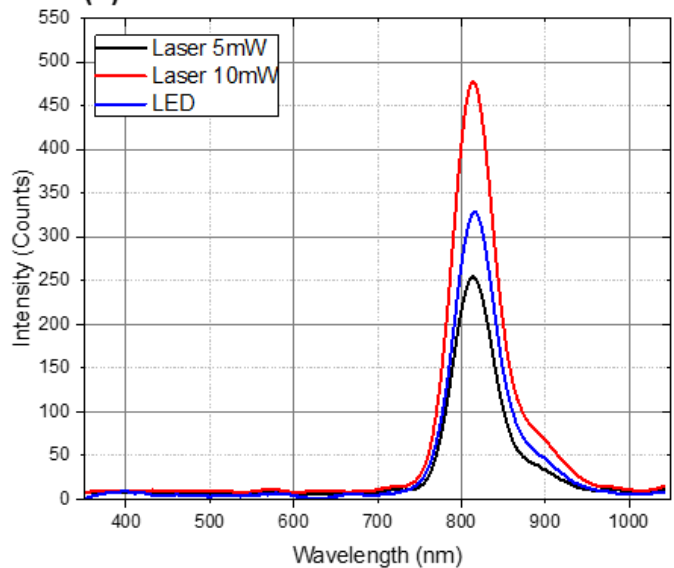

(d)

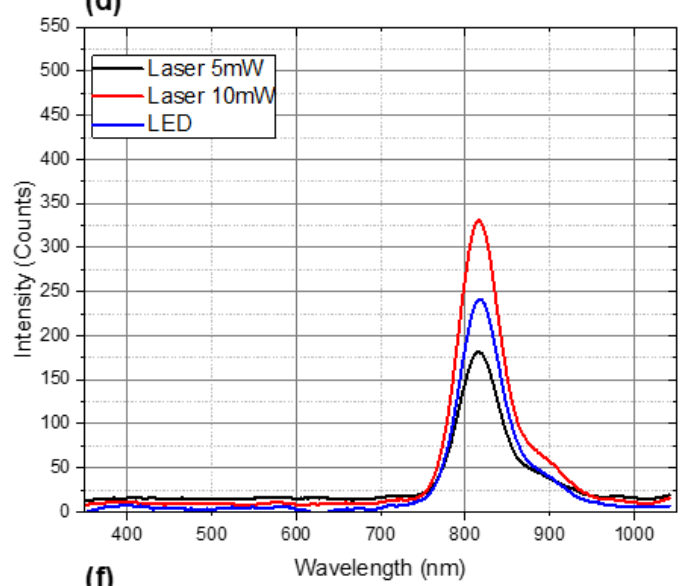

(f)

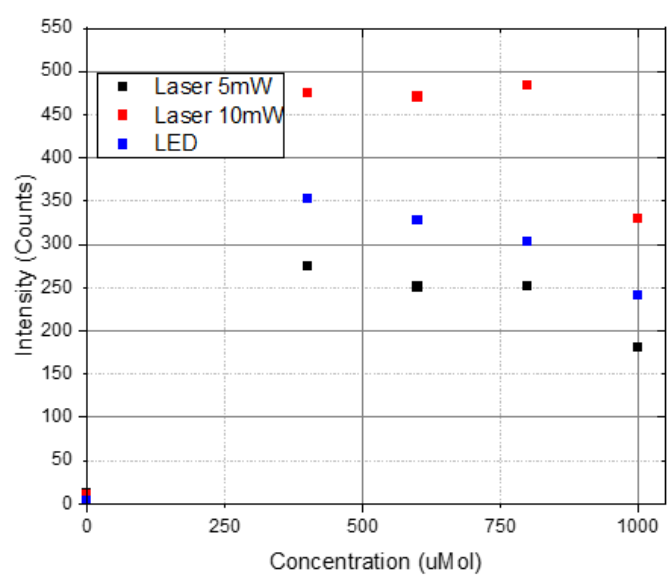

Figure. 4. Averaged and smoothed emission spectrometer data for a $400 \mu \mathrm{Mol}$ (a), $600 \mu \mathrm{Mol}$ (b), $800 \mu \mathrm{Mol}$ (c), $1000 \mu \mathrm{Mol}$ (d) solution of ICG:HSA, Water (e) as a reference and the peak power of fluorescence at $818 \mathrm{~nm}(\mathrm{f}), \mathrm{n}=6$ 
(a)

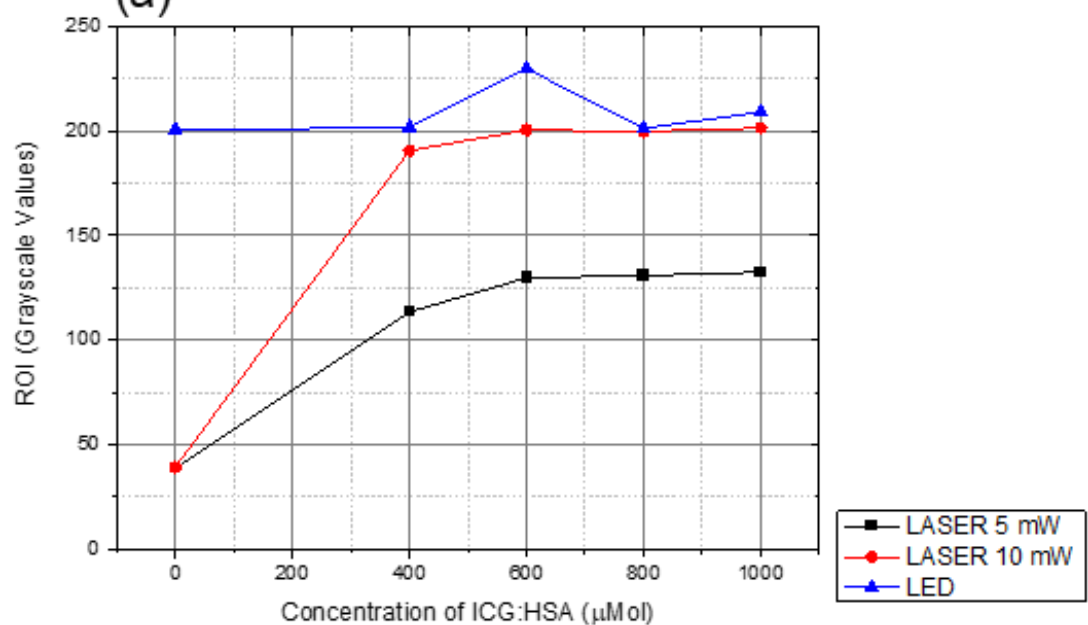

(b)

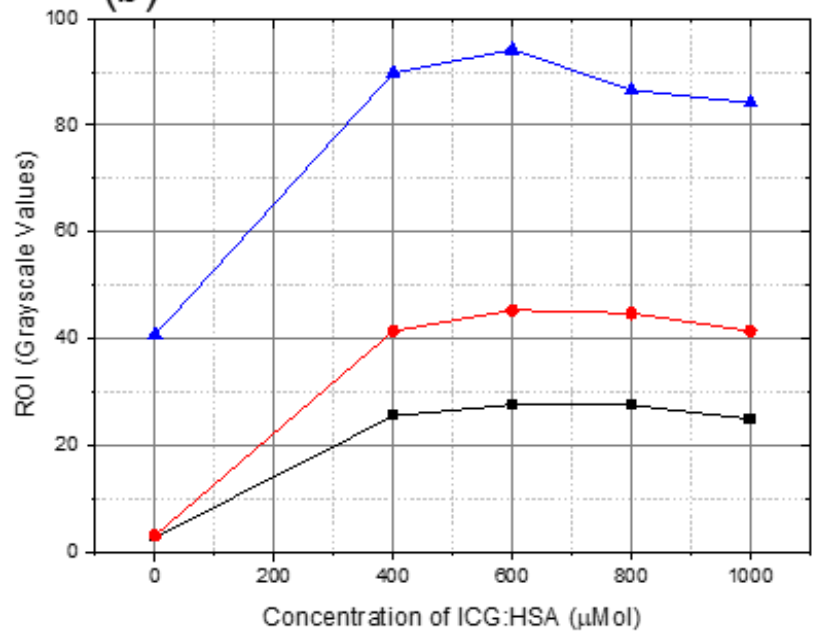

(c)

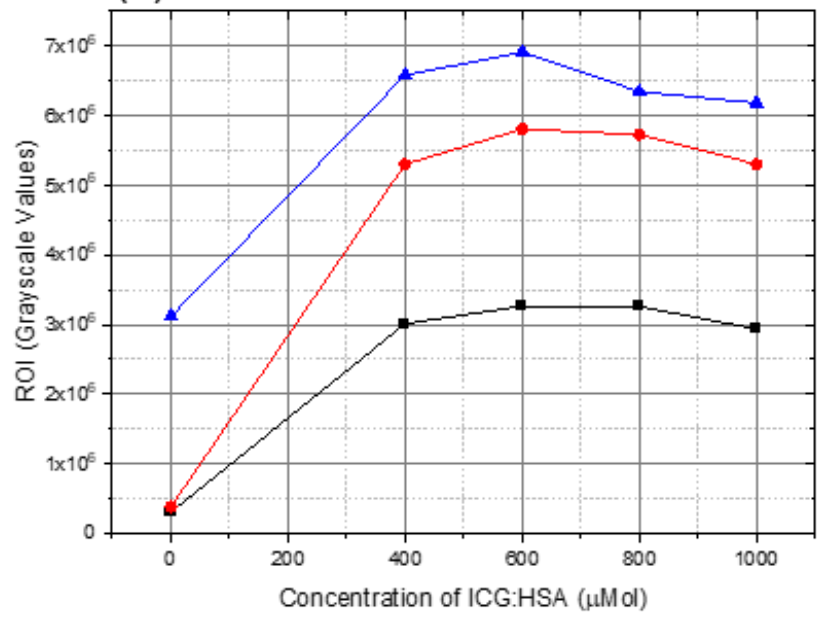

Figure 5. The maximum (a), mean (b), and total (c) intensity of pixels within the selected region for each concentration of ICG:HSA under each excitation condition, $\mathrm{n}=1$. Image captured at an exposure of $78124 \mu$ s and the ROI represents an average area of 107618 pixels 
(a)

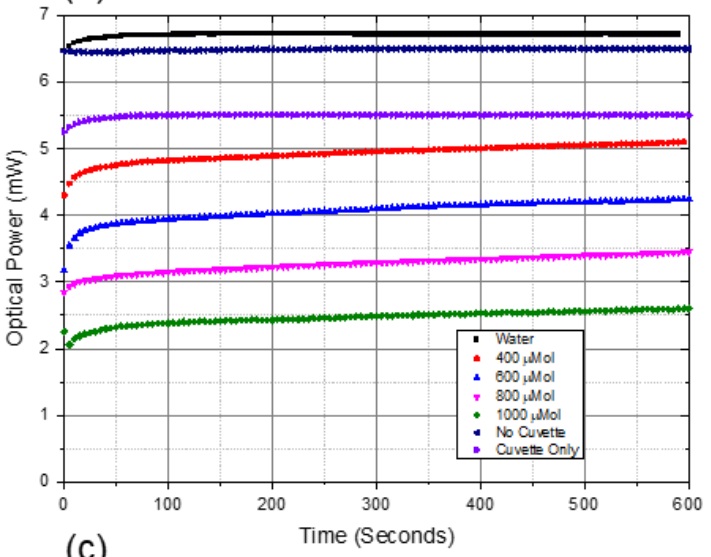

(c)

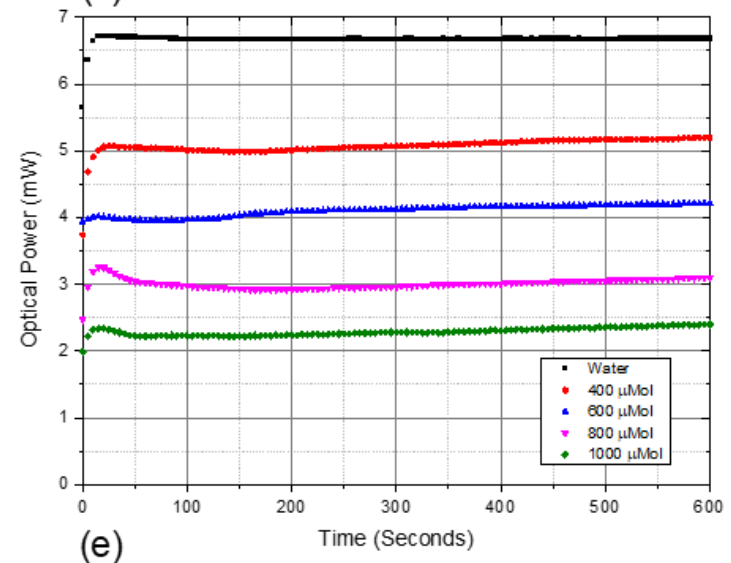

(e)

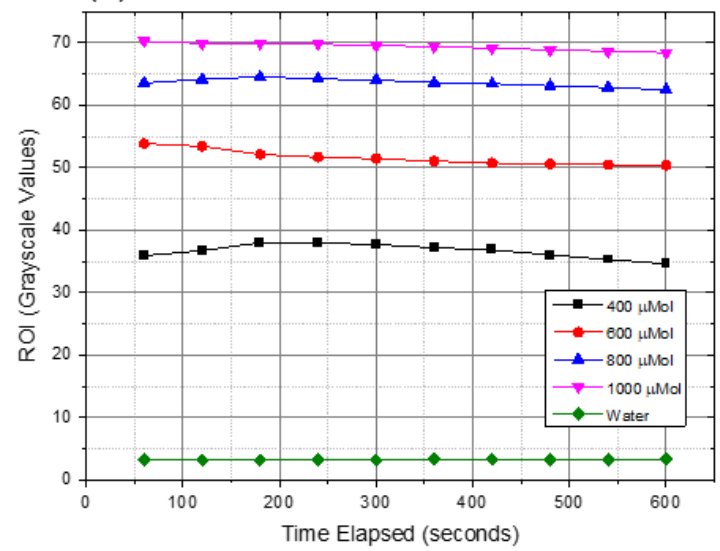

(b)

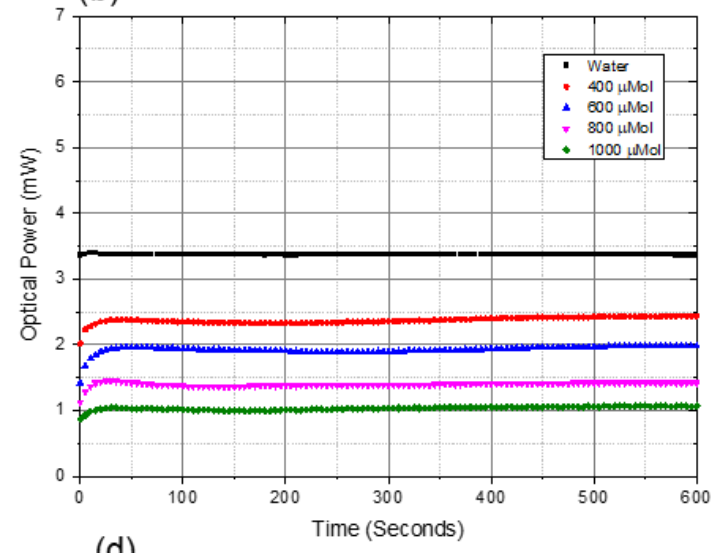

(d)

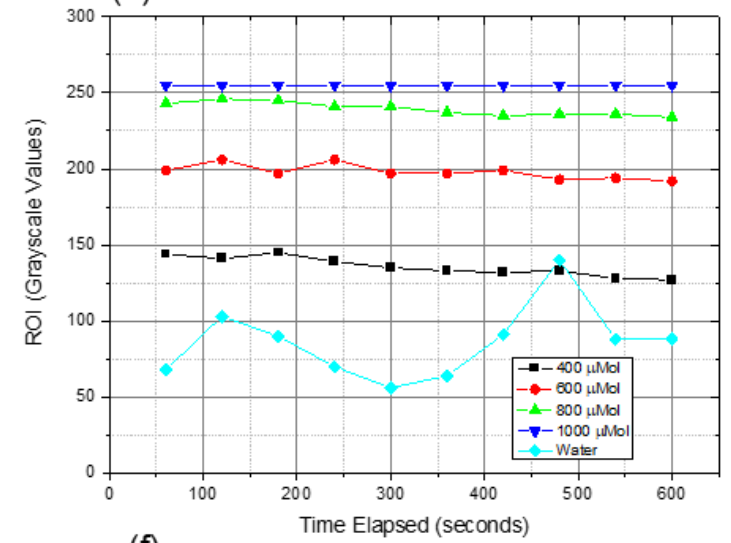

(f)

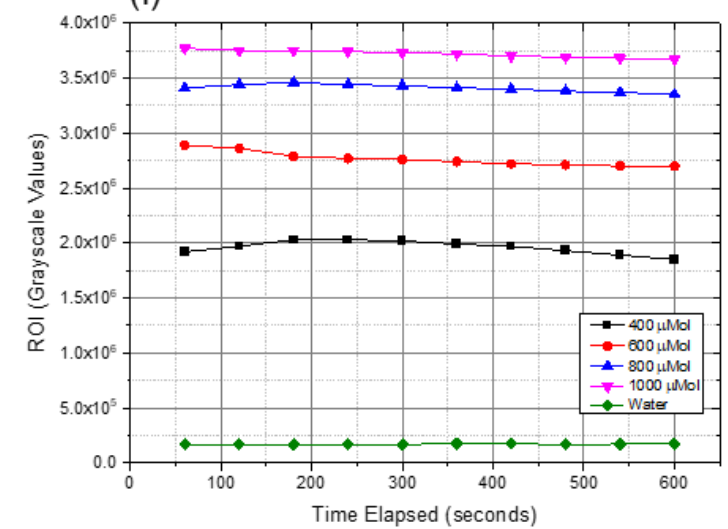

Figure 6: A plot showing the change in optical power with time using a power meter linear to the LED (a), 5mW LASER (b), and 10mW LASER (c), n=3; and maximum (d), mean (e), and total (f) intensity of pixels within the selected region for each concentration of ICG:HSA under $10 \mathrm{~mW}$ LASER excitation over a time period of 10 minutes, $\mathrm{n}=1$. The image was captured at an exposure of $124998 \mu$ s and the ROI represents an area of 53578 pixels

Figure 6(a), (b) and (c) show how the optical power changes with time for each excitation condition with a range of samples on test. The power meter detector is arranged in parallel with the excitation source so that excitation light passes through the sample be-fore detection. Optical power values were recorded every 5 seconds and plotted to determine the relationship between the cuvette contents, the optical power, the excitation source and time.
Figure 6(d), (e) and (f) present the maximum, mean and total pixel intensities respectively calculated in LabVIEW. Images are analyzed with a region of interest protocol as per the previous figure, Figure 5(a), (b) and (c) however, a string of images are analyzed in sequence in order to understand the relationship between the pixel intensity and time under excitation. With this data we can determine if there is any photobleaching present and which excitation condition and sample is most affected. 
Table 3: Degradation of the fluorescence ( $T=0$ minutes $\mathbf{T}=10$ minutes)

\begin{tabular}{|c|c|c|}
\hline Solution & $\begin{array}{c}\text { 10mW LASER } \\
\text { Excitation with } \\
\text { Camera } \\
\text { Perpendicular to } \\
\text { Source } \\
\text { (Grayscale values } \\
\text { per minute) }\end{array}$ & $\begin{array}{c}\text { 10mW LASER } \\
\text { Excitation with } \\
\text { Power Meter } \\
\text { Opposite the Source } \\
\text { (mW per minute) }\end{array}$ \\
\hline $400 \mu \mathrm{Mol}$ & 0.13 & -0.14 \\
\hline $600 \mu \mathrm{Mol}$ & 3.47 & -0.03 \\
\hline $800 \mu \mathrm{Mol}$ & 1.08 & -0.06 \\
\hline $1000 \mu \mathrm{Mol}$ & 1.84 & -0.04 \\
\hline Water & -0.10 & -0.10 \\
\hline
\end{tabular}

The results indicate degradation of the fluorescence at a varying rate as displayed in Table 3 , the mean pixel intensity values are used in column 2 taken from Figure 6(e) and optical power for column 3 taken from Figure 6(c). It is important to note the apparatus orientation as this can provide explanation as to why the power meter values are negative. The power meter is arranged opposite the source as seen in Figure 2 which would not necessarily indicate the rate of fluorescence decay. The optical power detected at the beginning of the experiment is less than that after an observation period of 10 minutes hence the negative values. It is reasonable to assume that over time the fluorescent material within the cuvette absorbs energy and will begin to photobleach once the threshold has been reached. More source energy is detected as absorption reduces following a reduction in available fluorophores, which also reduces the overall intensity of fluorescence. Source energy passing through the cuvette without absorption will be detected as the power meter cannot distinguish spectra. The power detected with the power meter is composed of fluorescence and source spectra. Without using a filter this cannot be verified but is an observation from experimental work as we have confirmed that fluorescence has also de-creased over time. No filter was used so as to simplify the system and to verify that a filter is not essential when using a single camera detection technique.

Temperature within the cuvette is recorded and the average temperature increase over a period of 11 minutes is presented in Table 4. The LASER is connected to a thermoelectric cooler in order to maintain the operating temperature for output stability, the LED is not connected to an active thermal management system during experiment as it is mounted in a custom aluminium holder. The differences in thermal management is likely to have a significant effect on temperature within the cuvette as shown in the following Table 4.
Table 4: Temperature within the cuvette

\begin{tabular}{|c|c|}
\hline Illumination & Temperature Change \\
\hline $5 \mathrm{~mW}$ LASER & $+0.13^{\circ} \mathrm{C}$ \\
\hline $10 \mathrm{~mW}$ LASER & $+0.30^{\circ} \mathrm{C}$ \\
\hline LED & $+0.60^{\circ} \mathrm{C}$ \\
\hline
\end{tabular}

A GPIO trigger was connected between the LED controller and the camera in order to synchronize data collection. The lowest framerate possible at $4855 \mu$ s was selected so that once the LED channel is set low, the camera channel is set high and an image is captured. The following figure (Figure 7) shows a schematic representation of the protocol followed whereby $\triangle \mathrm{K}$ is the time the LED is on, $\Delta \mathrm{T}$ is the time between the LED channel off and camera channel on and $\Delta \mathrm{S}$ is the exposure time, all are changeable parameters. No com-bination of testing conditions has resulted in any detectable fluorescence in the frame captured as soon as the excitation is switched off. This confirms that the ICG:HSA solutions on test do not have a lasting fluorescence decay that is detectable at frame rates of $199.98 \mathrm{~Hz}$ or lower. There is no present residual "glow effect" following excitation and therefore the fluorescent marker cannot be exploited by imaging it using a pulsed source and a corresponding negatively pulsed detector at a rate of $200 \mathrm{~Hz}$ or lower. This could have had a significant impact on patient safety during endoscopic diagnostics and therapy if viable. Literature indicates that femtosecond [20] and picosecond [21] pulse rates are appropriate for capturing ICG decay however, this system is limited by the human eye in regards to visible residual fluorescence. The cost of a high-speed camera system for fluorescence endoscopy would be exponentially higher than the proposed design and would not hold any value in real-world applications. If the fluorescence decay could have been detected via a camera capturing at millisecond speeds, then this would allow surgeons to visualize fluorescent material (diseased tissue) during endoscopic procedures in real-time.

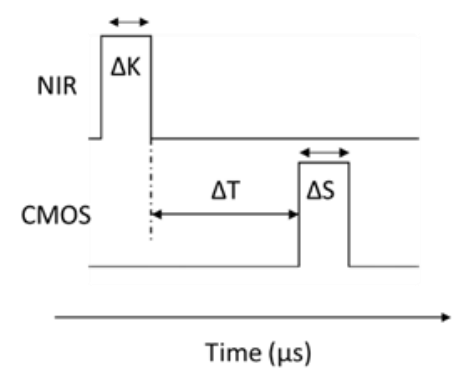

Figure 7: A schematic of the triggered pulses controlled via the Gardasoft LED controller with the NIR LED channel and the CMOS camera channel 


\section{DISCUSSION}

This research confirms that at an exposure time equivalent to $200 \mathrm{~Hz}$, there is no detectable residual fluorescence when a sample containing ICG in solution is excited at $780 \mathrm{~nm}$. If any residual fluorescence existed, then this could have a significant impact on medical imaging techniques such as minimally invasive fluorescence endoscopy as it would allow for a pulsed excitation. Pulsing the excitation would reduce the total time whereby the source is emitting, thus reducing the energy that the patient is exposed to during the procedure. Currently at least one NIR channel is mixed with a white light channel with both remaining on throughout the surgery. The risk of damage through excess heat is significantly higher when the channels remain on. Should there be any residual fluorescence in the moments following the excitation off, $\Delta \mathrm{K}=0$ then a faster exposure time should be used to detect its presence, $\Delta \mathrm{T}=0$ and $\Delta \mathrm{S}<4855 \mu \mathrm{s}$.

The experiments verify that fluorescence, generated from excitation of ICG in a solution, degrades with time during exposure to light at the excitation wavelength. The changes in fluorescence intensity observed during this study do not indicate a proportional relation-ship between concentration of ICG in solution and degradation of fluorescence. Data collected indicates that there are most likely secondary mechanisms occurring at a molecular level within the solution that have an effect on absorption and consequently on fluorescence.

The results show that there is a relationship between the rate of degradation of fluorescence and the optical power of the excitation source but again, this is not proportional. In general, as expected, the higher the optical power of the excitation, the quicker the rate of degradation of fluorescence. Temperature inside the cuvette was recorded for each excitation source. The LED recorded a temperature increase $50 \%$ higher than the $10 \mathrm{~mW}$ LASER and $80 \%$ higher than the $5 \mathrm{~mW}$ LASER however, this data does not suggest any proportional relationship with fluorescence decay. The thermal differences observed are likely to impact on changes in the solution at a molecular level which contributes to the overall effect of photodegradation. According to the experimental findings, after a period of 10 minutes there will a detectable difference in fluorescence intensity however, this difference is not significant and may not even be detectable to the naked eye.

Results from this experimental work verify that exposing a solution containing ICG to a $780 \mathrm{~nm}$ excitation source generates detectable fluorescence that cannot be seen by the naked eye. Over time, a reduction in fluorescence intensity is observed and this can be detected using either a power meter or an off-the-shelf CMOS camera. The camera used recorded a quantum efficiency of less than $30 \%$ at NIR wavelengths however, both fluorescent and non-fluorescent regions could be detected with good contrast. This refers to the ICG sample within the cuvette and the non-fluorescing background within the frame respectively. For liquid samples, the experimental setup is able to confirm the presence of ICG without the need for filters.

\section{CONCLUSIONS}

From this experimental work, the optical properties of ICG in solution are investigated using an excitation wavelength of $780 \mathrm{~nm}$. Apparatus including a single monochrome camera and LED controller are assembled for synchronized data collection, whereby aqueous solutions of ICG are exposed to controlled pulses of excitation wavelength. The main objective is to understand more about the optical properties of ICG in solution in or-der to exploit its properties and enhance its usage.

With the safety surrounding fluorescence endoscopy as the motivation for this work, significantly reducing energy exposure through pulsing of the NIR channel would result in an improvement in patient wellbeing. This experiment has confirmed that there is no detectable fluorescence postexcitation-pulse at frame rates of $200 \mathrm{~Hz}$ and lower with a slight delay in signal generation $(0.1 \mathrm{~ms})$. This indicates that in order to detect fluorescence at a frame rate of $200 \mathrm{~Hz}$, the source must always be present and subsequently filtered out of the frame. This research shows that at $780 \mathrm{~nm}$, both fluorescent and non-fluorescent regions of the frame can be detected using a single CMOS camera. This may be of benefit in future work as the excitation wavelength is visible to the naked eye but the fluorescence is not and this has been the most significant finding. If an accurate and effective method for detecting the presence of ICG in a sample is required, this can be accomplished using a cheap monochrome camera and an illumination source at $780 \mathrm{~nm}$. If fluorescence is pre-sent through the camera but not by eye then ICG is present.

With regards to photobleaching and photostability of ICG in solution, a variety of parameters and conditions have been investigated to demonstrate that there is no one parameter that proportionally affects the decrease in fluorescence intensity observed. Instead, it is likely that a combination of parameters influence the rate of fluorescence decay and some of which, should be considered at a molecular level.

\section{FUNDING}

Knowledge Economy Skills Scholarships (KESS) is a panWales higher level skills initiative led by Bangor University on behalf of the HE sector in Wales. It is part funded by the Welsh Government's European Social Fund (ESF) convergence programme for West Wales and the Valleys

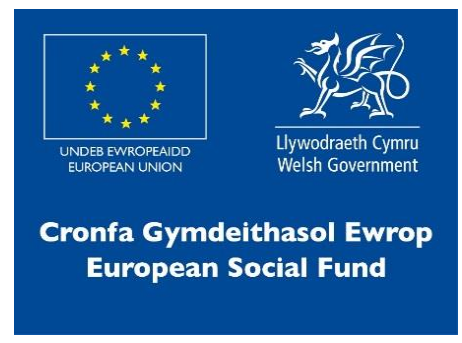

\section{ACKNOWLEDGEMENTS}

A special thank you to KESS and to the Faculty of Computing, Engineering and Science at the University of South Wales.

\section{CONFLICTS OF INTEREST}

The authors declare no conflict of interest. The funders had no role in the design of the study; in the collection, analyses, or interpretation of data; in the writing of the manuscript, or in the decision to publish the results. 


\section{REFERENCES}

[1] Alander, J.T.; Kaartinen, I.; Laakso, A.; Pätilä, T.; Spillmann, T.; Tuchin, V.V.; Venermo, M. and Välisuo, P. A Review of In-docyanine Green Fluorescent Imaging in Surgery. International Journal of Biomedical Imaging Volume 2012; 2012:940585.

[2] Lade, U.; Bhoyar, P. and Hingankar, M. Newly Developed Application of NIR Dyes in Cancer Targeting and Imaging: A Review. World Journal of Pharmacy and Pharmaceutical Sciences. 2014 Volume 3, Issue 2, 21932201

[3] Gibbs, S.L. Near infrared fluorescence for image-guided surgery. Quantitative Imaging in Medical Surgery 2012 Sep; 2(3): 177-187

[4] Jonak, C.; Skvara, H.; Kunstfeld, R.; Trautinger, F. and Schmid, J.A. Intradermal Indocyanine Green for In Vivo Fluorescence Laser Scanning Microscopy of Human Skin: A Pilot Study. PLoS ONE. 2011; 6(8):e23972

[5] Yaqoob, Z.; McDowell, E.; Wu, J.; Heng, X.; Fingler, J. and Yang, C. Molecular contrast optical coherence tomography: A pump-probe scheme using indocyanine green as a contrast agent. Journal of Biomedical Optics 2006, Sept-Oct; 11(5):054017

[6] Haj-Hosseini, N.; Behm, P.; Shabo, I. and Wårdell, K. Fluorescence spectroscopy using indocyanine green for lymph node mapping. Proceedings of SPIE, the international Society for Optical Engineering 2014, (8935), 893504, 1-6

[7] Frangioni, J. New Technologies for Human Cancer Imaging. American Society of Clinical Oncology. August 2008 Volume 26 Number 24

[8] Qi, J.; Nabavi, E.; Hu; Y.; Rees-Whippey, D.; Curtis, A.; Price, C.; Copner, N.; Sanassy, C.; Leiloglou, M.; Leff, D.; Hanna, G. and Elson, D. A light-weight near infrared fluorescence endoscope based on a single color camera: a proof-of-concept study. Conference on Lasers and Electro-Optics Pacific Rim (CLEO-PR) 31 July-4 Aug. 2017

[9] Kozin, E.D.; Lehmann, A.; Carter, M.; Hight, E.; Cohen, M.; Nakajima, H.H. and Lee, D.J. Thermal effects of endoscopy in human temporal bone model: implications for endoscopic ear surgery. Laryngoscope 2014 Aug: 124(8): E332-E339

[10] Modlin, I.M.; Begos, D.G. and Ballantyne, G.H. Laparoscopic Gastrointestinal Surgery: Current State of the Art. Edr. Spiro HM. Clinical Gastroenterology 2010

[11] Envemeka, C.S. Attenuation and penetration of visible $632.8 \mathrm{~nm}$ and invisible infra-red $904 \mathrm{~nm}$ light in soft tissues. LASER Therapy Volume 13, 2001 95-101

[12] Ohnishi, S.; Lomnes, S.; Laurence, R.; Gogbashian, A.; Mariani, G. and Frangioni, J. Organic Alternatives to
Quantum Dots for Intraoperative Near-Infrared Fluorescent Sentinel Lymph Node Mapping. Molecular Imaging Vo 4. No. 3 July 2005 pp 172-181

[13] Hutteman, M.; Mieog, J.; van der Voost, J.; Liefers, G.; Putter, H.; Löwik, C.; Frangioni, J.; van de Velde, C. and Vahrmeijer, A. Randomized, double-blind comparison of indocyanine green with or without albumin premixing for near-infrared fluo-rescence imaging of sentinel lymph nodes in breast cancer patients. Breast Cancer Research and Treatment. 2011 May; 127(1): 163-170

[14] Gioux, S.; Choi, H. and Frangioni, J. Image-Guided Surgery using Invisible Near-Infrared Light: Fundamentals of Clinical Translation. Molecular Imaging 2010 October; 9(5): 237-255

[15] Bozkurt, A. and Onaral, B. Safety assessment of near infrared light emitting diodes for diffuse optical measurements. Bio-medical Engineering OnLine 2004, 3:9

[16] Hachey, K.; Gilmore, D.; Armstrong, K.; Harris, S.; Hornick, J.; Colson, Y. and Wee, J. Safety and Feasability of Near Infrared Image-Guided Lymphatic Mapping of Regional Lymph Nodes in Esophogeal Cancer. Journal of Thoracic Cardiovascular Surgery. 2016 August; 152(2): 546-554

[17] Nairat, M.; Konar, A.; Kaniecki, M.; Lozovoy, V. and Dantus, M. Investigating the role of human serum albumin protein pocket on the excited state dynamics of indocyanine green using shaped femtosecond laser pulses. Royal Society of Chemistry PCCP 2015, 17, 5872

[18] Crull, J.W. and Schafer, S.A. Indocyanine Green Degradation During High-Intensity Laser Irradiation. SPIE 2671, Lasers in surgery: Advanced Characterization, Therapeutics, and Systems VI, (17 May 1996)

[19] Yuan, B.; Chen, N. and Zhu, Q. Emission and absorption properties of indocyanine green in Intralipid solution. Journal of Biomedical Optics 2004; 9(3): 497-503

[20] Gerega, A.; Zolek, N.; Soltysinski, T.; Milej, D.; Sawosz, P.; Toczylowska, B. and Liebert, A. Wavelengthresolved measure-ments of fluorescence lifetime of indocyanine green,” J. Biomed. Opt. 16(6), 067,010. 2011

[21] Homulle, H.A.R.; Powolny, F.; Stegehuis, P. L.; Dijkstra，J.; Li，D.U.; Homicsko, K.; Rimoldi, D.; Muehlethaler, K.; Prior, J.O.; Sinisi, R.; Dubikovskaya, E.; Charbon, E. and Bruschini, C. Compact solid-state CMOS single-photon detector array for in vivo NIR fluorescence lifetime oncology measurements. Biomedical Optics Express, 7(5), 1797-1814, 2016 